Check for updates

Cite this: RSC Adv., 2017, 7, 39814

Received 1st June 2017

Accepted 8th August 2017

DOI: 10.1039/c7ra06126a

rsc.li/rsc-advances

\section{Inserting AgCl@rGO into graphene hydrogel 3D structure: synergy of adsorption and photocatalysis for efficient removal of bisphenol $A \dagger$}

\author{
Fangyuan Chen, Junxiu Zhao, Weijia An, Jinshan Hu, Yinghua Liang \\ and Wenquan Cui (D) *
}

An AgClagraphene ( $r G O$ ) core-shell structure was fabricated and then loaded into reduced graphene oxide hydrogel $(\mathrm{rGH})$ to form AgClarGO-rGH by the chemical reduction method. The AgClarGO core-shell structure inhibited the aggregation of the $\mathrm{AgCl}$ particles and promoted the rapid transfer and separation of photogenerated electron-hole pairs. Moreover, the AgClarGO-rGH composite exhibited a high adsorption and photocatalytic degradation capacity for bisphenol A (BPA). Specifically, the degradation efficiency of BPA on AgClarGO-rGH-2 reached 93.7\% under the synergy of adsorption and photocatalytic degradation, and the degradation efficiency of BPA reached $87.0 \%$ after five cycles of degradation, which demonstrated the great synergistic effect between graphene and $\mathrm{AgCl}$. The degradation capabilities of AgClarGO-rGH were 6.4 and 2.8 times of pure $\mathrm{AgCl}$ and $\mathrm{rGH}$ on the synergistic degradation of BPA. In the continuous flow system, the degradation ratio of AgClarGO-rGH2 remained $100 \%$ within the first $4 \mathrm{~h}$ under the synergy conditions. When the reaction time reached $9 \mathrm{~h}$, the synergistic degradation ratio of BPA remained about $75.2 \%$. It showed that AgClarGO-rGH-2 still has good degradation activity and long life in the mobile phase system.

\section{Introduction}

In recent years, self-assembled graphene hydrogel has been considered one of the most promising methods in the "bottomup" nanotechnology and has subsequently become one of the most active areas of research in nanomaterials. ${ }^{1-3}$ Graphene composite hydrogels with three-dimensional porous structure possess many superior physical and chemical properties, such as high electronic and thermal conductivity, large theoretical surface area, great mechanical strength, and facile chemical functionalization. ${ }^{4,5}$ Since the macro-structure constructed with graphene nano modules contains continuously interconnected pores, the surface area of graphene hydrogel is 1-3 times of those of other types of graphene materials. ${ }^{6,7}$ Therefore, graphene hydrogels exhibit a high affinity to organic pollutants with dramatically enhanced adsorption capacity compared with the non-directional spread adsorption in graphene layers. ${ }^{8-10}$ In addition, 3D graphene hydrogel with mesoporous structure is very convenient to recycle and reuse, making this material ideal for adsorption purification. ${ }^{11}$

College of Chemical Engineering, Hebei Key Laboratory for Environment Photocatalytic and Electrocatalytic Materials, North China University of Science and Technology, Tangshan 063210, P. R. China. E-mail: wkcui@163.com; Tel: +86 315 8805460

† Electronic supplementary information (ESI) available: Supplementary materials for FTIR images, XPS, UV-vis, etc. See DOI: 10.1039/c7ra06126a
Photocatalytic degradation $^{\mathbf{1 2}}$ and adsorption $^{13}$ are two important means for removing toxic and harmful organic pollutants from water. In general, the photocatalytic oxidation reaction has very mild reaction conditions and complete mineralization. The adsorption process possesses various advantages, including simple operation procedure, convenient separation, and without secondary pollution. ${ }^{\mathbf{1 4}}$ Consequently, these two approaches have significant practical applications in solving environmental issues, especially in the aspect of water pollution. However, these two methods suffer some drawbacks, such as relatively slow mineralization rate, difficulty in recovering the photocatalytic material, slow adsorption ratio, and low capacity of adsorption. As a result, none of these two approaches alone will be able to meet and address the needs of various practical applications in the technical and economic aspects. ${ }^{15-17}$

Recently, constructing graphene hydrogels with threedimensional nanostructures has become a more common strategy to improve the properties of semiconductor compounds. ${ }^{18,19}$ For example, Sridhar Vadahanambi et al. successfully synthesized G-CNT- $\mathrm{Fe}_{2} \mathrm{O}_{3}$ hydrogel composite material using microwave-assisted in situ method. Their studies demonstrated that three dimensional structure of G-CNT- $\mathrm{Fe}_{2} \mathrm{O}_{3}$ could accelerate the diffusion of As(III) ions through the open pore network during the adsorption process, which led to a twofold increase in the adsorption capacity of $3 \mathrm{~d}$ G-CNT- $\mathrm{Fe}_{2} \mathrm{O}_{3}$ hydrogel compared with $2 \mathrm{~d}$ G- $\mathrm{Fe}_{2} \mathrm{O}_{3}{ }^{20}$ Similarly, Han et al. 
prepared CdS $/ \mathrm{P}_{25} / \mathrm{GH}$ composite material via a one-step hydrothermal and self-assembly method. They discovered that CdS and $\mathrm{P}_{25}$ nanoparticles were anchored in the mesoporous network of graphene hydrogels densely, which shorted the photoproduction electron transfer path effectively and promoted the separation of the electron-hole. As a result, the photocurrent intensity of compound increased 2.6 and 3.6 times than those of $\mathrm{CdS}$ and $\mathrm{P}_{25}$, respectively. ${ }^{21}$ From these results, it is evident that the three-dimensional graphene hydrogel materials showed good prospects for applications in the field of adsorption and photocatalysis.

$\mathrm{AgCl}$, an efficient photocatalyst, applied in combination with metal and photocatalytic characteristics of semiconductor, exhibits excellent photocatalytic performance. This property of $\mathrm{AgCl}$ has been confirmed by the studies from Huang etc. ${ }^{22,23}$ Under the visible light irradiation, $\mathrm{Ag}+$ could be reduced to Ag0 readily. AgCl has significant advantages in the degradation of organic pollutants because of the significant plasmon resonance effect of $\mathrm{Ag}$ and the apparent response to visible light of $\mathrm{AgCl} .^{24}$ Limited by easy aggregation, instability, small surface area, and recycle difficulty, $\mathrm{AgCl}$ is not practical in actual application. In previous work, although the prepared $\mathrm{AgCl}-$ activated carbon sample improves the dispensability and possesses the adsorption-photocatalytic synergy function, the adsorption sites of porous activated carbon do not agree with the photocatalytic sites of $\mathrm{Ag} @ A g \mathrm{Cl}$, which results in instability of sample activity. Recently, semiconductor composite catalyst with core-shell structure has attracted increasing research attention because of its superior performance, high stability of the catalyst with shell protection, improved photocatalytic stability, etc. ${ }^{25}$ On the other hand, it is well established that graphene oxide (GO) or reducing graphene oxide (rGO) can accelerate electrons and holes separation process in the photocatalytic reaction, thus improving the photocatalytic performance of the catalyst. ${ }^{26-28}$

Therefore, in this work, AgCl@rGO-rGH was successfully prepared by compositing the graphene hydrogels with $\mathrm{AgCl}$ via a chemical reduction method. In this process, AgCl nanoparticles were anchored on the sheet of graphene oxide and formed a three-dimensional network structure with mass reduced graphene oxide. The prepared AgCl@rGO-rGH sample exhibited the characteristics of surface adsorption and photocatalytic degradation of adsorbed organic matters, thus achieving the adsorption and photocatalytic synergy. Moreover, the rGH spatial three-dimensional macroporous structure provides convenient conditions for the recycling of photocatalytic composite. In addition, the adsorption, photocatalytic degradation, and adsorption photocatalytic synergy of bisphenol A (BPA) on the AgCl@rGO-rGH were investigated to improve light removal efficiency and the degradation efficiency of the catalyst.

\section{Experimental}

\subsection{Preparation of AgCl-rGH}

GO prepared by Hummer's method and Vitamin C (VC) (GO : $\mathrm{VC}=1: 10$, mass ratio) was mixed and ultra-sounded for
$6 \mathrm{~h}$. The resulting mixture was heated at $95{ }^{\circ} \mathrm{C}$ for $1-2$ hours. Then, the collected product was washed with distilled water after it was immersed in water for several hours to completely remove the excessive unreacted VC. After washed several times, the product was transferred to a beaker and stirred until it was smashed up. Subsequently, an appropriate amount of silver ammonia solution was added to the water mixture containing the prepared GO and stirred for $30 \mathrm{~min}$, and then one equivalent of $\mathrm{NaCl}$ aqueous solution was added and stirred for additional $2 \mathrm{~h}$. After the reaction, the resulting product was washed with deionized water several times and dried by vacuum freezedrying.

\subsection{Preparation of rGO wrapped AgCl graphene hydrogel composites}

Multi-layer GO wrapped AgCl graphene hydrogel composites. A small amount GO prepared by Hummer's method was ultra-sounded for $8 \mathrm{~h}$. An appropriate amount of silver ammonia solution was added to deionized water and stirred for $30 \mathrm{~min}$, and then a $\mathrm{NaCl}$ aqueous solution was added to the prepared silver ammonia solution and stirred for $4 \mathrm{~h}$. To the above prepared $\mathrm{AgCl}$ mixture was added GO and VC, in dropwise ( $\mathrm{GO}: \mathrm{VC}=1: 10$, mass ratio) ultrasounded for $6 \mathrm{~h}$, and heated at $95{ }^{\circ} \mathrm{C}$ for $1-2$ hours. After the reaction, the resulting product was washed with deionized water several times and dried by vacuum freeze-drying. This sample was named $\mathrm{AgCl} @ \mathrm{rGO}-\mathrm{rGH}-1$. Preparation of less-layer GO wrapped AgCl graphene hydrogel composites was same as preparation of AgCl@rGO-rGH-1 except that a small amount of GO prepared by Hummer's method was ultrasounded for $6 \mathrm{~h}$ in $\mathrm{pH}=10$ buffer solution. This sample was named AgCl@rGO-rGH-2. The overall process for the fabrication of the AgCl-rGH, AgCl@rGO-rGH-1 and $\mathrm{AgCl} @$ rGO-rGH-2 is shown in Fig. 1.

\subsection{Photocatalyst characterization}

$\mathrm{X}$-ray diffractometry (XRD) was performed using a Rigaku D/MAX2500 PC diffractometer with $\mathrm{Cu} \mathrm{K} \alpha$ radiation at an operating voltage of $40 \mathrm{kV}$ and an operating current of $100 \mathrm{~mA}$. The morphologies of the prepared samples were examined by transmission electron microscopy (TEM) using a Hitachi HT 7700 electron microscope operating at an accelerating voltage of $100 \mathrm{kV}$. High-resolution transmission electron microscopy (HRTEM) images were obtained using a JEM2100 field emission transmission electron microscope with an accelerating voltage of $200 \mathrm{kV}$. UV-vis light (UV-vis) diffuse reflectance spectra were recorded on a UV-vis spectrometer (Puxi, UV1901). Fourier transform infrared (FT-IR) spectra were collected using Bruker V70FTIR spectrometer in the frequency range of $4000-500 \mathrm{~cm}^{-1}$ with a resolution of $1 \mathrm{~cm}^{-1}$. X-ray photoelectron spectroscopy (XPS) was performed on a PHI 5300 ESCA system. The beam voltage was $3.0 \mathrm{kV}$, and the energy of the Ar ion beam was $1.0 \mathrm{keV}$. The binding energies were normalized to the signal for adventitious carbon at $284.8 \mathrm{eV}$. Electrochemical and photoelectrochemical measurements were performed in a threeelectrode quartz cell with $0.1 \mathrm{M} \mathrm{Na}_{2} \mathrm{SO}_{4}$ electrolyte solution. A platinum wire was used as the counter electrode, and 


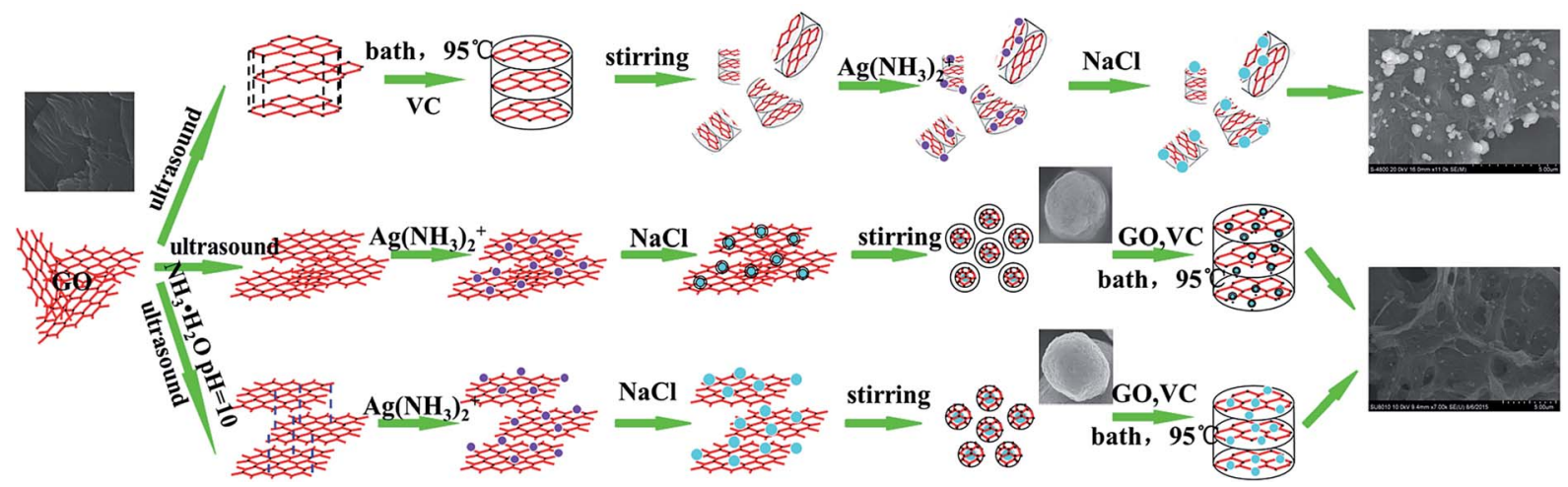

Fig. 1 Schematic illustration of synthesis of $\mathrm{AgCl}-\mathrm{rGH}, \mathrm{AgCl}(\mathrm{arGO}-\mathrm{rGH}-1$ and AgClarGO-rGH-2.

a saturated calomel electrode (SCE) was used as the reference electrode. Sample $\mathrm{lm}$ electrodes on ITO served as working electrodes. The photoelectrochemical experimental results were obtained with an electrochemical system (CHI-660D, China).

\subsection{Synergy of photocatalysis and adsorption in the degradation of BPA}

Static activity test. A $250 \mathrm{~W}$ halide lamp (Philips) with a $420 \mathrm{~nm}$ cut-off filter was used at a distance of $10 \mathrm{~cm}$ from an unsealed beaker. The temperature was maintained at $25 \pm 2{ }^{\circ} \mathrm{C}$. For each test, $0.1 \mathrm{~g}$ catalyst powder was added to $100 \mathrm{~mL} 10 \mathrm{ppm}$ BPA solution. The concentration of the BPA in the solution phase was determined by UV-vis spectrophotometer and HighPerformance Liquid Chromatography (HPLC). The maximum absorption wavelength of BPA at lambda $=278 \mathrm{~nm}$ was used as the HPLC detection wavelength. The detailed test process is as follows: adsorption performance: an equivalent amount of catalyst $(0.1 \mathrm{~g}$ of each $\mathrm{AgCl}$ composite materials prepared by different methods, and the equivalent amount of $\mathrm{AgCl}-\mathrm{rGH}$ $(\mathrm{AgCl}(0.095 \mathrm{~g})$ and $\mathrm{rGH}(0.005 \mathrm{~g}))$ were added to the $100 \mathrm{~mL}$ $10 \mathrm{ppm}$ BPA solution. A $3 \mathrm{~mL}$ aliquot of the suspension mixture was taken at intervals and filtered through a filter membrane $(0.45 \mu \mathrm{m})$ before the determination of concentration by using HPLC. Adsorption-photocatalysis performance: $0.1 \mathrm{~g}$ catalyst powder was added to $100 \mathrm{~mL} 10 \mathrm{ppm}$ BPA solution. Before the irradiation, the test solution was stirred in the dark for $30 \mathrm{~min}$ to reach the adsorption equilibrium and then exposed to the light. A $3 \mathrm{~mL}$ aliquot of the suspension mixture was taken at intervals, and filtered through a filter membrane $(0.45 \mu \mathrm{m})$ before the determination of concentration. Photocatalytic degradation saturated adsorption liquid: $0.1 \mathrm{~g}$ photocatalysts was added into the $100 \mathrm{~mL} 10 \mathrm{ppm}$ BPA without irradiation. After adsorption equilibrium, these photocatalysts are re-added to another $100 \mathrm{~mL} 10 \mathrm{ppm}$ BPA and directly exposed to the light for photocatalysis. Photocatalytic degradation primary liquid test is consistent with adsorption-photocatalysis test. However, $C_{0}$ is the concentration after adsorption equilibrium in the calculation of $C / C_{0}$. The synergy of photocatalysis and adsorption: $0.1 \mathrm{~g}$ catalyst powder was added to $100 \mathrm{~mL} 10 \mathrm{ppm}$ BPA solution and directly exposed to the light for synergy reaction. A $3 \mathrm{~mL}$ aliquot of the suspension mixture was taken at intervals and filtered through a filter membrane $(0.45 \mu \mathrm{m})$ before the determination of concentration by using HPLC.

The stability test of AgCl@rGO-rGH-2: $0.1 \mathrm{~g}$ sample was added to, $100 \mathrm{~mL} 10 \mathrm{ppm}$ BPA solution for recycling purification in different conditions, including adsorption, photocatalytic degradation saturated adsorption liquid and synergy of photocatalysis and adsorption for $60 \mathrm{~min}$. The sample was not cleaned or dried in each recycle reaction. A $3 \mathrm{~mL}$ aliquot of the suspension mixture was taken at intervals and filtered with a filter membrane $(0.45 \mu \mathrm{m})$ before the determination.

Continues flow reaction. Fig. 2 illustrates the test system for the dynamic activity. The xenon lamp (Model: CHF-XW500) was used as the light source, and a $420 \mathrm{~nm}$ cutoff filter was used at a distance of $5 \mathrm{~cm}$ from the reactor to avoid the influence of UV light. The reactor dimension was $40 \mathrm{~mm} \times 20 \mathrm{~mm} \times 2 \mathrm{~mm}$, and the concentration of BPA was $10 \mathrm{ppm}$ with a flow rate of $0.2 \mathrm{~mL} \mathrm{~min}^{-1} . C_{0}$ is initial concentration and $C$ is the concentration from the outlet liquid in the calculation of $C / C_{0}$. The flow rate of the BPA solution was controlled by a peristaltic pump. At given intervals, the reaction mixture was sampled with a syringe, filtered, and analyzed by using spectrophotometer and HPLC testing. The maximum absorption wavelength of BPA at lambda $=278 \mathrm{~nm}$ was used as the detection wavelength. In detail: adsorption performance: $0.5 \mathrm{~g}$ catalyst was immobilized in the reactor without irradiation. At intervals, the degraded solution at the outlet and was determined by HPLC. Photocatalytic performance: $0.5 \mathrm{~g}$ catalyst was immobilized in the reactor without irradiation. When the concentration of the

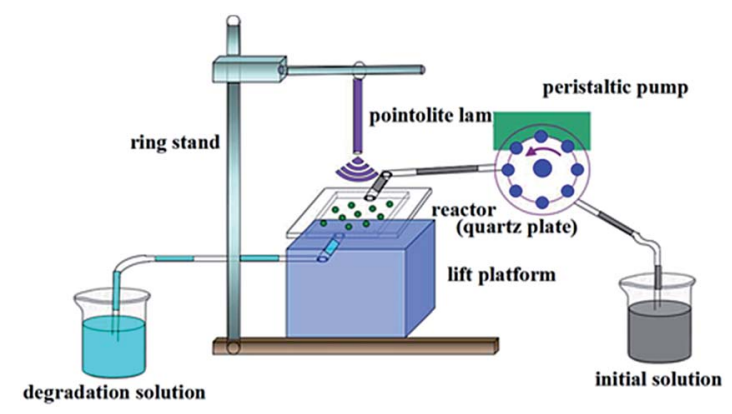

Fig. 2 The device of dynamic photocatalytic test system. 
solution is consistent with the initial concentration, the lamp was turned on and the concentration of the solution is measured at interval. Synergy of adsorption-photocatalysis: $0.5 \mathrm{~g}$ catalyst was immobilized in the reactor and directly turned on. At intervals, the degraded solution at the outlet and was determined by HPLC.

\section{Result and discussion}

\subsection{Characterization of catalysts}

Fig. 3a-c showed the SEM images of GO, rGH, and $\mathrm{AgCl}$, respectively. From these images, the morphology of GO exhibited a layer structure with a significant aggregation, which dramatically reduced the surface area of GO. In contrast, Fig. $3 \mathrm{~b}$ reveals that $\mathrm{rGH}$ was composed of branches with honeycomb shapes and assembled lamella GO to three-dimensional net structure, thus providing more contact sites for $\mathrm{AgCl}$ nanoparticles. AgCl was observed as spherical-like particles with particle size ranging from 1 to $3 \mu \mathrm{m}$, which is not well controllable and could aggregate easily.

Fig. 3d-f display the images of AgCl-(5 wt\%)rGH, AgCl@rGO(5 wt\%)rGH-1, and AgCl@rGO-(5 wt\%)rGH-2, respectively, were prepared by different methods. AgCl-(5 wt\%)rGH was prepared by the impregnation method. Fig. 3d indicates that $\mathrm{Ag}$ and $\mathrm{AgCl}$ nanoparticles scattered disorderly on graphene layer surface or interlamination, in which $\mathrm{AgCl}$ particles had a relatively large size of approximately $400 \mathrm{~nm}$ while the size of $\mathrm{Ag}$ particles was in the range of 10 to $50 \mathrm{~nm}$. Because the AgCl generation
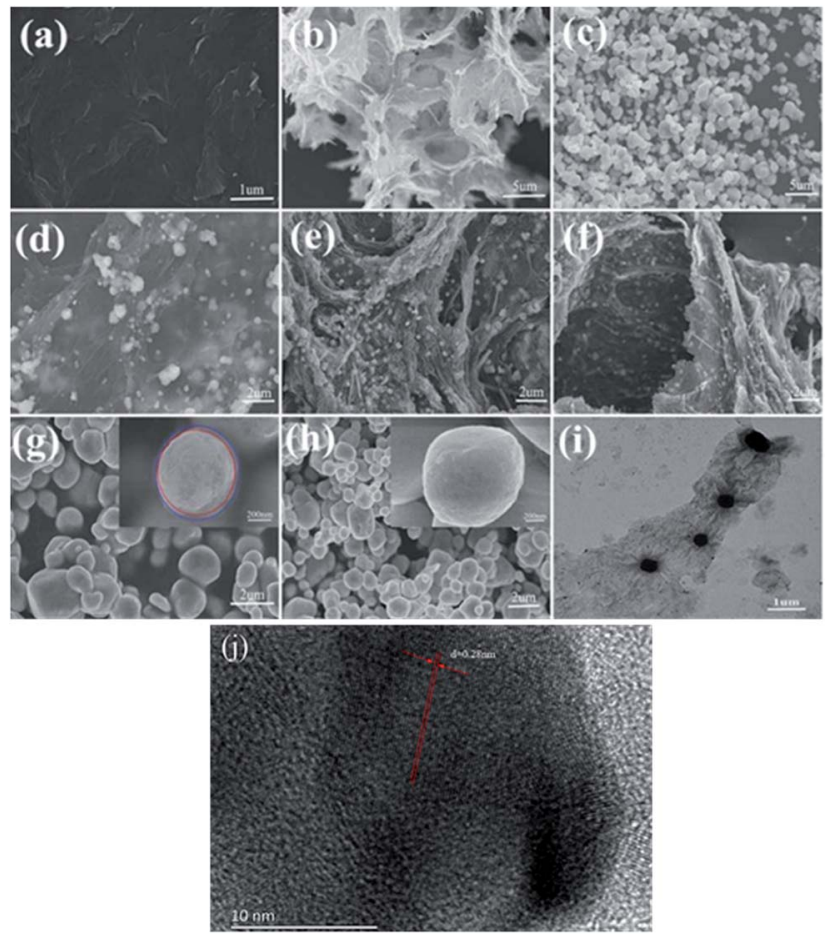

Fig. 3 SEM images of (a) GO, (b) $\mathrm{rGH}$, (c) pure $\mathrm{AgCl}$, (d) $\mathrm{AgCl}-(5 \mathrm{wt} \%)$ rGH, (e) AgClarGO-(5 wt\%)rGH-1，(f) AgClarGO-(5 wt\%)rGH-2, (g) AgClaGO-1, (h) AgClaGO-2, (i) TEM of AgClaGO-2 and (j) HRTEM of AgClarGO-(5 wt\%)rGH-2. process is after the formation of graphene hydrogels, the morphology and particle size of $\mathrm{AgCl}$ are not controlled. Different than AgCl-(5 wt\%)rGH, AgCl@rGO-(5 wt\%)rGH-1 and AgCl@rGO-(5 wt\%)rGH-2 were prepared by the chemical reduction method. Since the $\mathrm{AgCl}$ particles in these two materials were pre-coated with small amounts of GO, the structure of AgCl@GO core-shell and the morphology and stability of the $\mathrm{AgCl}$ were controlled to some degree. When a significant amount of $\mathrm{GO}$ and $\mathrm{AgCl} @ \mathrm{GO}$ mixed, the pre-coated $\mathrm{AgCl}$ could not only avoid the excessive reduction by $\mathrm{VC}$ but also control the neighboring graphene hydrogels by extrusion effect. As a result, as the film of $\mathrm{AgCl}$ and main skeleton structure of composite material, GO played a key role in controlling morphology, space filling, direction, and supporting effect. $^{29,30}$

Fig. $3 \mathrm{~g}$ and $\mathrm{h}$ show the SEM images of AgCl@GO treated with different methods. Fig. 3g shows SEM image of AgCl@GO-1, in which GO was only subjected to the ultrasonic treatment. It was observed that almost each $\mathrm{AgCl}$ particle was coated with a thin film. The thickness of the film was determined to be about 20-30 nm according to the inset in Fig. 3g. GO in AgCl@GO-2 was treated by alkali $(\mathrm{pH}=10)$ and ultrasonic treatments. ${ }^{31}$ As shown in Fig. $3 \mathrm{~h}$, this combined treatment not only reduced the size of GO shell but also separated GO layers and reduced the amount of GO adhered on the surface of the AgCl. From the inset in Fig. 3h, GO was hard to be observed on the surface of the AgCl. Therefore, the shading effect of graphene could be significantly reduced, and the photocatalytic degradation performance of composite material AgCl@GO-2 could be dramatically improved. Fig. 3i displays the TEM image of the precursor of AgCl@GO-2, in which the sample dispersed evenly with a size of approximately $300 \mathrm{~nm}$. Fig. $3 \mathrm{j}$ of indicated the lattice spacing of $\mathrm{AgCl}(0.28 \mathrm{~nm})$ is good agreement with the crystal planes for the $\left(\begin{array}{lll}2 & 0 & 0\end{array}\right)$ face. In addition, $\mathrm{AgCl}$ and $\mathrm{rGH}$ are in close contact from the Fig. $3 \mathrm{j} .{ }^{32}$

Fig. 4 displayed the XRD patterns of $\mathrm{rGH}, \mathrm{AgCl}, \mathrm{AgCl}-\mathrm{rGH}$, AgCl@rGO-rGH-1, and AgCl@rGO-rGH-2. The characteristic peak of rGH was clearly observed at $24.8^{\circ}$. The diffraction peaks at $27.9^{\circ}, 32.3^{\circ}, 54.8^{\circ}$, and $57.5^{\circ}$ corresponded to (111), (200), (311), (222) crystal planes of $\mathrm{AgCl}$, respectively. ${ }^{32}$ The diffraction peaks at $38.1^{\circ}$ and $44.0^{\circ}$ corresponded to (111) and (200) crystal

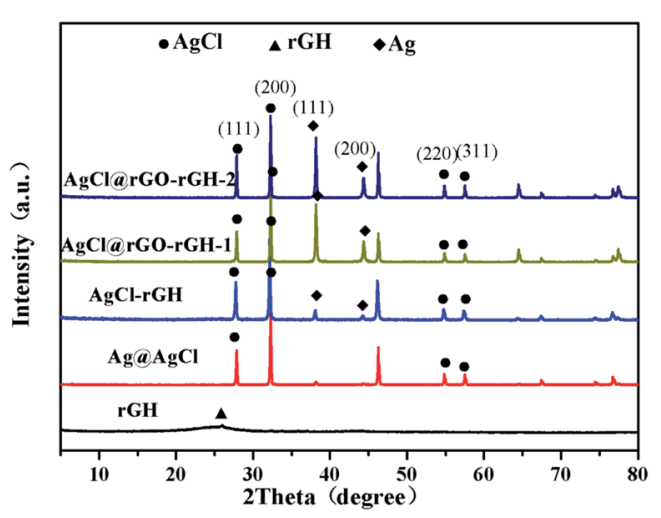

Fig. 4 XRD patterns of the different materials. 
planes of Ag, respectively. As shown in Fig. 4, the characteristic peaks of $\mathrm{AgCl}$ in all composites' hydrogels composites were clearly observed, which demonstrated that all the methods for the preparation of the composite were successful. However, it was found that the characteristic peaks of $\mathrm{Ag}$ in the sample prepared by the chemical reduction method exhibited stronger intensity, which is attributed to the excessive VC in this preparation procedure. On the other hand, compared with $\mathrm{rGH}$, the characteristic peak of $\mathrm{rGH}$ at $24.8^{\circ}$ was not observed in all prepared composites, which is due likely to the existence of a large amount of $\mathrm{AgCl}$ that fill the space of $\mathrm{rGH}$ and to a minor content of rGH. Quantitative analysis and purity identification were confirmed by infrared spectra ${ }^{33}$ (Fig. S1†).

X-ray photoelectron spectroscopy (XPS) analysis of AgCl@rGO-rGH-2, GO, and rGH were carried out to reveal more detail information about their element compositions and chemical states. ${ }^{34}$ Fig. S2† presents the XPS survey spectra of AgCl@rGO-rGH-2, GO, and rGH, in which the carbon and oxygen functional groups of different composites were observed. ${ }^{35} \mathrm{C} / \mathrm{O}$ ratio obtained from XPS roses from 2.9 to 4.9 after being reduced ( $\mathrm{GO}$ to $\mathrm{rGH}$ ), and from 4.9 to 5.8 after joining $\mathrm{AgCl}$ (rGH to AgCl@rGO-(5 wt\%)rGH-2), which demonstrates that the chemical reduction method is an effective reduction method, but also indicates that the addition of $\mathrm{AgCl}$ may deepen the degree of reduction. ${ }^{31,36}$ Fig. $S 2 d \dagger$ represents the $\mathrm{Ag} 3 \mathrm{~d}$ region of the $\mathrm{AgCl}$ and $\mathrm{AgCl} @ \mathrm{rGO}-(5 \mathrm{wt} \%) \mathrm{rGH}-2$. This may be due to that the addition of $\mathrm{VC}$ caused a part of the $\mathrm{Ag}+$ reduction to form Ag0 in the formation of the complex, causing Ag3d of the composite shifted to a higher binding energy. ${ }^{37}$

Fig. 5a displayed the UV-vis diffuse reflectance spectra of $\mathrm{AgCl}$ graphene hydrogels composites prepared by different methods. From the Fig. 5, these three composites had the same ratio of $\mathrm{rGH}$, but all three curves in the figure appeared different degree of red shifts (AgCl-rGH is weaker, AgCl@rGO-rGH-1 and AgCl@rGO-rGH-2 are stronger). The reason for this phenomenon is that there is a certain amount of $\mathrm{AgCl}$ nanoparticles located on the exterior of AgCl-(5 wt\%)rGH which is not loaded or coated on the $\mathrm{rGH}$ skeleton for AgCl-(5 wt\%)rGH, resulting in the loss of a large part of $\mathrm{AgCl}$ nanoparticles and weakening the absorption of visible light. The samples of AgCl@rGO-rGH-1 and AgCl@rGO-rGH-2, AgCl nanoparticles are neater than the former because of the protection of rGO, making the composite materials inherit the properties of $\mathrm{AgCl}$ and have a strong response to visible light. In addition, since all three composite materials contained a large amount of $\mathrm{Ag}$ nanoparticles, they exhibited significant plasmon resonance effect. $^{32}$ Fig. S3† showed the UV-vis diffuse map specific analysis of AgCl@rGOrGH-2 composite materials containing different ratios of $\mathrm{rGH}$. It was found that the light absorption of AgCl@rGO-rGH-2 increased with increasing ratio of rGH. However, the response range of visible light decreased from 540 to $420 \mathrm{~nm}$, indicating that the shading effect of rGH is very strong, even the ratio of $\mathrm{rGH}$ is relatively low.

Fig. S4† showed the Raman spectra of AgCl@rGO-(5 wt\%) $\mathrm{rGH}-2$, GO, and rGH composites with an excitation of $524 \mathrm{~nm}$ laser light. These samples exhibited two strong peaks, which were ascribed to the disorder peak (D, centered at $1350 \mathrm{~cm}^{-1}$ )
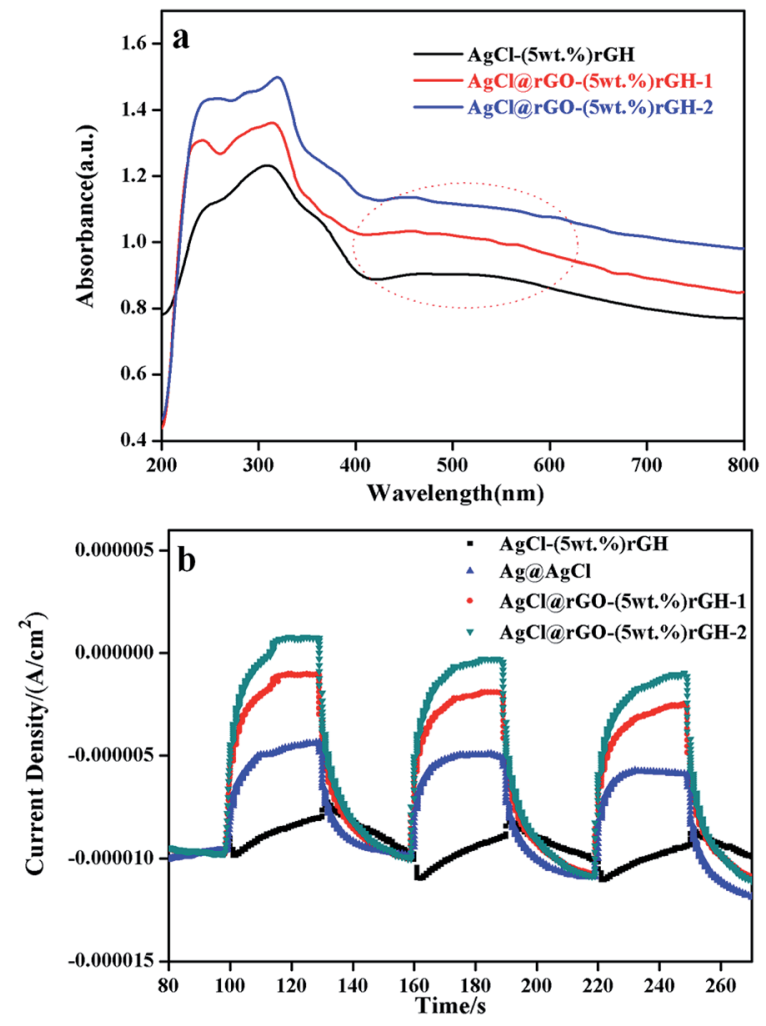

Fig. 5 (a) UV-vis diffuse reflectance spectra; (b) the photocurrent spectra of different materials.

and the graphitic peak (G, at $1580 \mathrm{~cm}-1) .{ }^{38}$ Compared with the $I_{\mathrm{D}} / I_{\mathrm{G}}$ value of $\mathrm{GO}$ and $\mathrm{rGH}$, the $I_{\mathrm{D}} / I_{\mathrm{G}}$ value of AgCl@rGO-rGH-2 increased, suggesting that the AgCl@rGO-rGH-2 produced more defects in carbon structure. ${ }^{39}$ Which could be caused by the strong interactions between $\mathrm{Ag}$ and $\mathrm{rGH}, \mathrm{AgCl}$ and $\mathrm{rGH}^{33}$ This 2D-band has been widely used to identify the number of graphene layers. D $+\mathrm{D}^{\prime}$ and $2 \mathrm{D}$ was observed at $2900 \mathrm{~cm}^{-1}$ and $2700 \mathrm{~cm}^{-1}$. A sharp symmetric peak corresponds to less-layer graphene whereas broad peaks are associated with multi-layer grapheme. ${ }^{40,41}$ The in-plane crystallite size $\left(L_{\mathrm{a}}\right)$ can be calculated by Tuinstra-Koenig (TK) relation $^{42}$ (eqn (S1) $\dagger$ ). La of AgCl@rGO-(5 wt\%)rGH-2, rGH, and GO were estimated to be $19.4,18.1$, and $16.4 \mathrm{~nm}$, which is associated with increase of content of defects. ${ }^{43}$

To investigate the photoelectrochemical properties of these samples, a three-electrode electrochemical setup was used to measure the photocurrent response. Fig. 6 showed the transient photocurrent responses of pure AgCl, AgCl-rGH, AgCl@rGOrGH-1, and AgCl@rGO-rGH-2. Among them, the photocurrent of AgCl@rGO-(5wt\%)rGH-2 was the highest, followed by the AgCl@rGO-(5 wt\%)rGH-1. In addition, most of AgCl nanoparticles in AgCl-rGH are large and scatter on the surface of rGH. Consequently, the photoelectron transfer path in AgClrGH is longer than AgCl@rGO core-shell, and the photocurrent intensity of $\mathrm{AgCl}-\mathrm{rGH}$ is the minimum. The photocurrent of rGH (5 wt $\%$ ) prepared by impregnation method was smaller than that of monomer $\mathrm{AgCl}$, which could be attributed to the adverse effect of AgCl-rGH composite on the separation of 


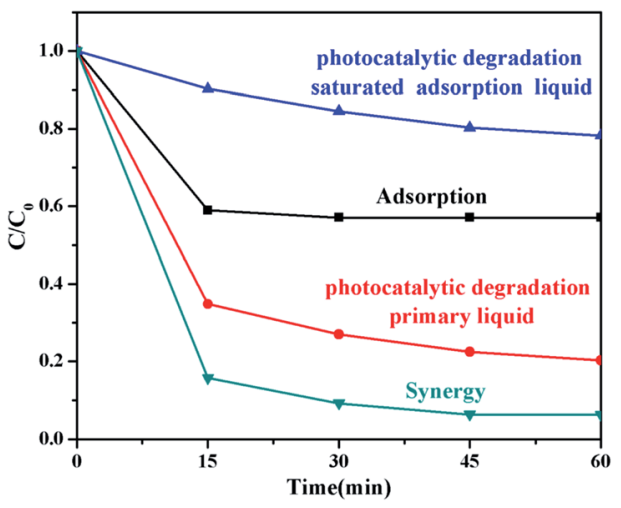

Fig. 6 Comparison of activities by different conditions with $0.1 \mathrm{~g}$ AgClarGO-(5 wt\%) rGH-2.

electrons and holes. Although these composites contain the same ratio of $\mathrm{rGH}$, their photocurrent intensity varied considerably. The reason for this phenomenon is that the separation degree of photogenerated electron-hole pair in these composites is different due to their different structures. ${ }^{25,44}$ Compared with AgCl@rGO-rGH-1, the shell of AgCl@rGO-rGH-2 is thinner and the light transmission is higher because of the addition of ammonia during the preparation. For this reason, the photocurrent intensity of AgCl@rGO-rGH-2 is the highest among three materials. Fig. S5† showed a typical EIS of the prepared samples, which clearly indicates that the diameter of the arc radius on the EIS Nyquist plot of the AgCl@rGO-(5wt\%)rGH-1 and AgCl@rGO-(5 wt\%)rGH-2 electrodes were much smaller than those of the $\mathrm{AgCl}-(5 \mathrm{wt} \%) \mathrm{rGH}$ and $\mathrm{AgCl}$ electrodes under visible light irradiation. This result implied that the AgCl@rGO(5 wt $\%$ )rGH-1 and AgCl@rGO-(5 wt\%)rGH-2 photoelectrodes exhibited the fastest interfacial charge transfer and the most efficient separation of photogenerated charge carriers. Moreover, AgCl@rGO-(5 wt\%)rGH-2 was treated with ammonia solution during the preparation process, which caused the $\mathrm{rGH}$ film to be separated to some degree. As a result, AgCl@rGO$(5 \mathrm{wt} \%) \mathrm{rGH}-2$ has a relatively smaller electric resistance and a shorter electron transfer path. ${ }^{45}$

\subsection{Synergy of adsorption-photocatalysis activity}

Whether can remove organic pollutants to a great extent is one of the most important indicators for purification activity of the composite material. Fig. 6 displays the removal ratio of BPA $(10 \mathrm{ppm}, 100 \mathrm{~mL})$ by pure adsorption, photocatalytic degradation saturated adsorption liquid (which means that these photocatalysts are re-added to another $100 \mathrm{~mL} 10 \mathrm{ppm}$ BPA and directly exposed to the light for photocatalysis after adsorption equilibrium), photocatalytic degradation primary liquid (which means that these photocatalysts are added to $100 \mathrm{~mL} 10 \mathrm{ppm}$ BPA solution. Before the irradiation, the test solution was stirred in the dark for $30 \mathrm{~min}$ to reach the adsorption equilibrium and then exposed to the light. $C_{0}$ in this represents the concentration at equilibrium, and adsorption-photocatalysis synergy with $0.1 \mathrm{~g}$ AgCl@rGO-(5 wt\%) rGH-2. The degradation ratio by synergy function was $93.7 \%$, which was greater than the sum rate $(64.7 \%)$ of pure adsorption and photocatalytic degradation of primary liquid. This result fully demonstrated the superiority of synergy effect. It was also found that the photocatalytic degradation removal ratio of adsorption equilibrium liquid was $79.0 \%$, whereas the removal ratio of the photocatalytic degradation primary liquid was $21.7 \%$ under the same conditions. This is mainly because the effect of adsorption of composites was not considered during the calculation of degradation removal ratio of adsorption equilibrium liquid. Although photocatalytic degradation experimental conditions were optimized, samples can inevitably adsorb BPA molecules when photocatalytic effect took place, thus increasing the adsorption quantity on the surface of the composite. Therefore, the actual photocatalytic degradation ratio is far less than $21.7 \%$. The photocatalytic degradation ratio is only about $10.9 \%$ if we assume that the ratio of photocatalytic degradation and adsorption is $1: 1$. Synergistic adsorption-photocatalytic degradation of pollutants to pollutants was adsorbed onto the surface of composites hydrogels at first, followed by degradation of pollutants, realized the synergistic effect of rapid adsorption enrichment and mineralization depth of oxidation. However, when the composites reached the adsorption equilibrium, photocatalytic degradation may be affected because the active sites are occupied. ${ }^{46,47}$

Fig. 7a displayed saturated adsorption amount of BPA (10 ppm, $100 \mathrm{~mL}$ ) by AgCl graphene hydrogel composite materials prepared by different methods, and an equivalent amount of $\mathrm{AgCl}(0.095 \mathrm{~g}), \mathrm{rGH}(0.005 \mathrm{~g})$. It can be seen from the Fig. 7a that the adsorption quantities of all materials except $\mathrm{AgCl}$ increased rapidly with increasing time, especially in the initial 10 minutes. After that, the adsorption qualities of all materials tended to saturation when the reaction occurred in $30 \mathrm{~min}$. In addition, the saturated adsorption capacities of $\mathrm{AgCl}, \mathrm{rGH}, \mathrm{AgCl}-$ (5 wt\%)rGH, AgCl@rGO-(5 wt\%)rGH-1, and AgCl@rGO-(5 wt\%) rGH-2 were $10.8 \%, 16.7 \%, 34.7 \%, 41.3 \%, 43.0 \%$, respectively. This result could be attributed to the microporous structure of rGH that provides enough channels for the adsorption process, which ensure the adsorbate BPA to reach the interior of composite quickly, thus substantially reducing the saturated adsorption time..$^{48}$ Compared to pure $\mathrm{AgCl}$ and $\mathrm{rGH}$, the specific surface areas of all composites have a certain improvement, although they are prepared by different methods. The increased specific surface areas of AgCl@rGO-(5 wt\%)rGH-1 and $\mathrm{AgCl} @ \mathrm{rGO}-(5 \mathrm{wt} \%) \mathrm{rGH}-2$ are ascribed to the interaction of $\mathrm{AgCl}$ and $\mathrm{rGH}$. During the formation of these two composites, $\mathrm{AgCl}$ might provide more efficient adsorption sites on the surface of rGH but also separate the layers of rGH. Fig. $7 \mathrm{~b}$ represented degradation of BPA by different materials. The adsorption reached equilibrium after $60 \mathrm{~min}$, followed by light degradation. These results are consistent with Fig. 6 and 7a. AgCl@rGO(5 wt\%)rGH-1 and AgCl@rGO-(5 wt\%)rGH-2 showed better degradation than other complexes.

Fig. 7c displayed the photocatalytic degradation of BPA $(10 \mathrm{ppm}, 100 \mathrm{~mL}$ ) after the adsorption equilibrium on $\mathrm{AgCl}$ graphene hydrogel composite material prepared by different methods and an equivalent amount of $\mathrm{AgCl}(0.095 \mathrm{~g})$. As shown in Fig. 7c, the AgCl@rGO-(5 wt\%)rGH-2 degradation effect is 



Fig. 7 (a) BPA adsorption capacity; (b) the adsorption-photocatalytic degradation of BPA; (c) the photocatalytic degradation of BPA; (d) the synergistic absorption-photocatalysis capabilities with varies materials. best, AgCl@rGO-(5 wt\%)rGH-1 followed, AgCl-(5 wt\%)rGH and $\mathrm{Ag} @ \mathrm{AgCl}$ worst. For the composites AgCl@rGO-(5 wt\%)rGH-1 and AgCl@rGO-(5 wt\%)rGH-2, AgCl is wrapped by rGH tightly. The AgCl@rGO core-shell structure can not only increase the stability of $\mathrm{AgCl}$ by preventing the reduction of $\mathrm{AgCl}$ to $\mathrm{Ag}$ under visible light irradiation but also facilitate the transfer of electrons produced by $\mathrm{AgCl}$ to the surface of rGH thus shortening the electron transfer path, reducing the recombination probability of electron-hole pairs, and improving the photocatalytic activity. Compared to AgCl@rGO-(5 wt\%)rGH-1, the rGH shell of AgCl in AgCl@rGO-(5 wt\%)rGH-2 was treated with ammonia solution during the preparation process, which separated the rGH film to some degree. Therefore, the light transmission and the photocatalytic degradation of AgCl-core was better than those of AgCl@rGO-(5 wt\%)rGH-1. In the case of composite $\mathrm{AgCl}-(5 \mathrm{wt} \%) \mathrm{rGH}$, most of the $\mathrm{AgCl}$ particles are anchored on the surface of $\mathrm{rGH}$, whcih would convert to $\mathrm{Ag}$ nanoparticles under visible light irradiation. In addition, the $\mathrm{AgCl}$ size in $\mathrm{AgCl}-(5 \mathrm{wt} \%) \mathrm{rGH}$ is bigger than those of AgCl@rGO-(5 wt\%) rGH-1 and AgCl@rGO-(5 wt\%)rGH-2, which results in a longer photoproduced electron transfer path in $\mathrm{AgCl}-(5 \mathrm{wt} \%) \mathrm{rGH}$, thus reducing the photocatalytic activity. For pure rGH, it does not have any photocatalytic activity because its opacity prevents the transmission of visible light. ${ }^{49}$

Fig. 7d displayed the synergistic degradation capability of BPA (10 ppm, $100 \mathrm{~mL}$ ) on AgCl graphene hydrogel composite materials prepared by different methods and an equivalent amount of $\mathrm{AgCl}(0.095 \mathrm{~g})$ and $\mathrm{rGH}(0.005 \mathrm{~g})$. Although the adsorption capability of rGH is strong, it hardy transmits visible light. Therefore, the synergy of $\mathrm{rGH}$ is equivalent to the adsorption effect of $\mathrm{rGH}$, and their synergistic degradation ratio was $33 \%$. Since the adsorption capability of $\mathrm{AgCl}$ is weak, it depends on its excellent photocatalytic degradation capability to remove pollutants. ${ }^{32}$ For this reason, the synergy degradation ratio of $\mathrm{AgCl}$ must be close to its photocatalytic degradation ratio, which is consistent with the experimental results. Although the morphology and structure of AgCl-rGH are not very ideal due to the preparation method, AgCl-rGH composite possesses excellent adsorption and photocatalytic performance, including adsorption ratio of $35.6 \%$, photocatalytic degradation ratio of $79.7 \%$, and synergy degradation ratio of $85.1 \%$.

Compared to AgCl-rGH, AgCl particles in AgCl@rGO-rGH-1 and AgCl@rGO-rGH-2 are protected by rGO during the preparation processes. The morphologies and structures of $\mathrm{AgCl}$ nanoparticles in AgCl@rGO-rGH-1 and AgCl@rGO-rGH-2 are better than those of AgCl-rGH, which results in their significant synergy effects. Since the $\mathrm{rGO}$ shell of $\mathrm{AgCl}$ is thinner in AgCl@rGO-rGH-2 than that of AgCl@rGO-rGH-1, the visible light is able to transmit more efficiently in AgCl@rGO-rGH-2. As a result, both the photocatalytic degradation and synergy degradation ratio of AgCl@rGO-rGH-2 are much better.

Fig. $\mathrm{S}^{\dagger} \uparrow$ showed the performance of different AgCl@rGO( $5 \mathrm{wt} \%$ )rGH-2 composites in the degradation of BPA from the water solution for the purpose of comparison of the adsorption and photocatalytic degradation and synergy performance. It turned out that the synergistic effects of different ratios of 
AgCl@rGO-rGH-2 composites are much higher than those of adsorption or photocatalysis only.

Fig. 8 showed the performance of the stability of AgCl@rGO(5 wt\%)rGH-2 with adsorption, photocatalytic degradation saturated adsorption liquid and synergy of photocatalysis and adsorption for $60 \mathrm{~min}$. It was found that only adsorption reaction occurred in the composite, in which the first time adsorption ratio of BPA was $43.0 \%$ while the adsorption ratio tended to become zero after three cycles of reaction. This result implied that the adsorption sites were occupied by BPA that could not be further removed. When only photocatalytic degradation reaction occurred in the composite, the photocatalytic degradation ratio of pollutant were $79.8 \%$ and $72.1 \%$ for the first time and the last time, respectively, in which the variation is not very significant. In the adsorption-photocatalysis synergistic process, although the degradation ratio of BPA slightly decreased after each cycle run (from $93.7 \%$ to $87.0 \%$ ), the final degradation ratio of $87.0 \%$ in adsorptionphotocatalysis synergistic process was higher than the degradation ratio of $80.1 \%$ in the first run of photocatalytic degradation only process. These results indicated that adsorption as a means of treatment of environmental pollutants only adsorbs the target pollutants on the surface of composite materials, which cannot completely degrade the pollutants. ${ }^{50}$ In contrast, the degradation ratio of pollutants in the photocatalytic degradation reaction process did not change significantly after multiple reaction cycles. The degradation ratio only decreased by $8 \%$ after five cycles of reaction, which could be attributed to the reduction of bare $\mathrm{AgCl}$ particles in the composite to $\mathrm{Ag}$ particles under visible light irradiation. Consequently, the synergy of adsorption and photocatalysis significantly improved the reusability and maintained the performance of AgCl@rGO(5 wt\%)rGH-2 under continuous working conditions. In addition, a slight erosion of the composite is one of the reasons that degradation ratio reduced after multiple cycles of reaction.

From a practical perspective, we examined the degradation activity of the composite in the continuous working conditions. The setup of mobile phase apparatus is shown in Fig. 2. Fig. 9a showed the degraded properties of $10 \mathrm{ppm}$ of BPA solution by $0.5 \mathrm{~g}$ AgCl@rGO-(5 wt\%)rGH-2 in liquid phase via simple

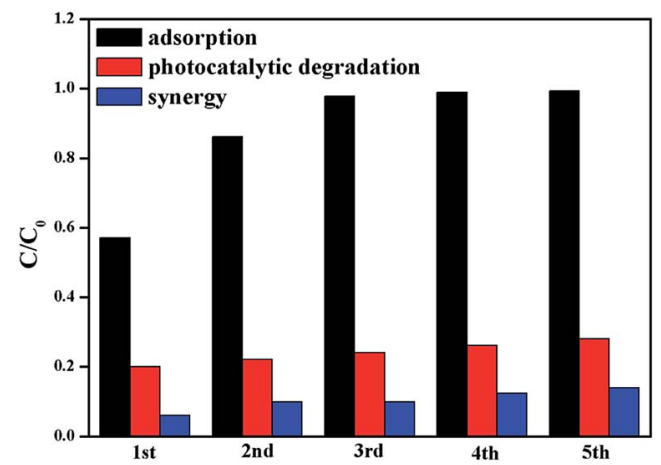

The cycle times of AgCl@rGO-rGH-2 in different conditions

Fig. 8 The stability of AgClarGO-(5 wt\%)rGH-2 in different conditions.


Fig. 9 (a) Comparison of activities of BPA (10 ppm) by different

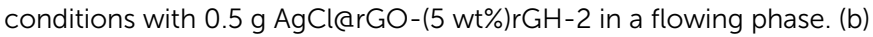
Adsorption property; (c) pure photocatalytic property with $0.5 \mathrm{~g}$ different ratio $\mathrm{AgCl}$ (arGO-rGH-2 in flowing phase.

adsorption, photocatalytic degradation, and adsorption-photocatalysis synergy. The degradation ratio of BPA was $100 \%$ within the first $3 \mathrm{~h}$ through the simple adsorption while the degradation ratio of BPA remained $100 \%$ within the first $4 \mathrm{~h}$ in the synergy process. After $9 \mathrm{~h}$ reaction, the BPA degradation efficiency started to stable at about $75.2 \%$. From this result, the synergy composite is more favourable in the degradation of BPA. From this result, the synergy composite is more favorable in the degradation of BPA. Composite only in the process of synergy adsorbed BPA for $1 \mathrm{~h}$ more than in adsorption or photocatalytic degradation process, which also illustrated that adsorption played a significant role in the degradation of BPA.

Fig. 9b displayed the adsorption property of BPA (10 ppm) by different ratios AgCl@rGO-rGH-2 composites. As shown in Fig. 9b, the ratio of rGH in the composites significantly affected the breakthrough points of composites. In the presence of $3 \%$, $5 \%$, and $7 \%$ ratios of $\mathrm{rGH}$, the breakthrough points of these composites were 2 h, 3 h, and 4 h, respectively. The AgCl@rGO- 
rGH-2 composite materials with $3 \%, 5 \%$, and $7 \%$ ratios of $\mathrm{rGH}$ were completely saturated after $27 \mathrm{~h}, 33 \mathrm{~h}$, and $38 \mathrm{~h}$, respectively. These results showed that with increasing ratio of $\mathrm{rGH}$, adsorption performance of composites enhanced significantly, and composites exhibited superior adsorption performance in the continuous flow conditions. Although the difference in the ratio of rGH in the composites is slight, the results of degradation performance varied markedly, which may be caused by the great specific surface of $\mathrm{rGH}^{48}$ Fig. 9c displayed photocatalytic properties of BPA (10 ppm) by AgCl@rGO-rGH-2 with different ratios of rGH. It is worth noting that the composites reached the saturation absorption before the saturation photocatalytic degradation. Moreover, the photocatalytic performance improved with the decrease of rGH content from 7 to $3 \mathrm{wt} \%$, which is associated with the shading effect of rGH. After $7 \mathrm{~h}$, the photocatalytic degradation ratio of BPA remained constant, which could be caused by the reduction of $\mathrm{AgCl}$ to $\mathrm{Ag}$ in the composite. Based on the characterization and the analysis of the active data of the above photocatalysts, the possible mechanism of photocatalytic degradation was proposed. The possible photocatalytic degradation mechanism is that when $\mathrm{Ag}$ and $\mathrm{AgCl}$ are excited to produce electrons $\left(\mathrm{e}^{-}\right)$and holes $\left(\mathrm{h}^{+}\right)$ when composite is irradiated by visible light. $\mathrm{e}^{-}$from $\mathrm{Ag}$ to $\mathrm{AgCl}$ and then to $\mathrm{rGH},{ }^{33}$ which could reacted with $\mathrm{O}_{2}$ to form ${ }^{\circ} \mathrm{O}^{2-}$. And ${ }^{\circ} \mathrm{O}_{2}{ }^{-}$and $\mathrm{h}^{+}$could degrade BPA. ${ }^{51}$ Thus, $\mathrm{e}^{-}$and $\mathrm{h}^{+}$can be effectively separated.

\section{Conclusions}

In summary, a novel AgCl@rGO-rGH with three-dimensional (3D) network structure was fabricated by the simple chemical reduction method. The SEM images of AgCl@GO core@shell composite showed that the prepared AgCl@rGO-rGH contained significant graphene oxide, which efficiently inhibited the reduction of $\mathrm{AgCl}$ particles to $\mathrm{Ag}$ particles, thus improving the efficient separation and transfer of photogenerated electronhole pairs. Moreover, AgCl@rGO-rGH exhibited superb performance for the degradation of BPA from aqueous solutions under different conditions. The degradation efficiency of BPA on AgCl@rGO-rGH-2 reached 93.7\% under the conditions of adsorption-photocatalysis synergy, which was mainly attributed to AgCl@rGO that significantly shortened the electron transfer path in the composite. Furthermore, after five cycles of degradation, the degradation efficiency of BPA reached $87 \%$, while the adsorption performance tended to become zero after five cycles of degradation. After $9 \mathrm{~h}$ reaction, the BPA degradation efficiency started to stable at about $75.2 \%$. The degradation activities of composites in static and continuous flow conditions demonstrated that AgCl@rGO-rGH-2 composite exhibited superior degradation activity and longer catalyst life in the continuous flow system.

\section{Conflicts of interest}

There are no conflicts to declare.

\section{Acknowledgements}

This work was financially supported by the National Natural Science Foundation of China (No. 51672081), Key Program of Natural Science of Hebei Province (B2016209375), Hebei Natural Science Funds for the Joint Research of Iron and Steel (B2016209348), Hebei Provincial Foundation for International cooperation (15391403D).

\section{Notes and references}

1 G. Mogilevsky, O. Hartman, E. D. Emmons, A. Balboa, J. B. DeCoste, B. J. Schindler, I. Iordanov and C. J. Karwacki, ACS Appl. Mater. Interfaces, 2014, 6, 1063810648.

2 J. J. Huang, W. X. Liu, L. Wang, X. M. Sun, F. W. Huo and J. F. Liu, Langmuir, 2014, 30, 4434-4440.

3 W. L. Yang, A. Lucotti, M. Tommasini and W. A. Chalifoux, J. Am. Chem. Soc., 2016, 138, 9137-9144.

4 J. C. Meyer, A. K. Geim, M. I. Katsnelson, K. S. Novoselov, T. J. Booth and S. Roth, Nature, 2007, 446, 60-63.

5 D. A. Reddy, J. Choi, S. Lee, R. Ma and T. K. Kim, RSC Adv., 2015, 5, 67394-67404.

6 S. Nardecchia, D. Carriazo, M. L. Ferrer, M. C. Gutierrez and F. D. Monte, Chem. Soc. Rev., 2013, 42, 794-830.

7 R. K. Upadhyay, N. Soin and S. S. Roy, RSC Adv., 2014, 4, 3823-3851.

8 W. J. Liu, J. Y. Cai, Z. X. Ding and Z. H. Li, Appl. Catal., B, 2015, 174, 421-426.

9 D. Kumar, A. Lee, T. Lee, M. Lim and D. K. Lim, Nano Lett., 2016, 16, 1760-1767.

10 Y. Zhuang, F. Yu, J. H. Chen and J. Ma, J. Environ. Chem. Eng., 2016, 4, 147-156.

11 Y. Shen, Q. Fang and B. L. Chen, Environ. Sci. Technol., 2014, 49, 67-84.

12 D. Ravelli, D. Dondi, M. Fagnonia and A. Albini, Chem. Soc. Rev., 2009, 38, 1999-2011.

13 J. Zhao, Z. Y. Wang, J. C. White and B. S. Xing, Environ. Sci. Technol., 2014, 48, 9995-10009.

14 P. N. Moza, K. Fytianos, V. Samanidou and F. Korte, Bull. Environ. Contam. Toxicol., 1988, 41, 678-682.

15 Z. Y. Sui, Y. Cui, J. H. Zhu and B. H. Han, ACS Appl. Mater. Interfaces, 2013, 5, 9172-9179.

16 G. X. Zhao, L. Jiang, Y. D. He, J. X. Li, H. L. Dong, X. K. Wang and W. P. Hu, Adv. Mater., 2011, 2, 3959-3963.

17 Y. Parent, D. Blake, K. Magrini-Bair, C. Lyons, C. Turchi, A. Watt, E. Wolfrum and M. Prairie, Sol. Energy, 1996, 56, 429-437.

18 W. J. Liu, J. Y. Cai and Z. H. Li, ACS Sustainable Chem. Eng., 2015, 3, 277-282.

19 D. A. Reddy, J. Choi, S. Lee, R. Ma and T. K. Kim, , RSC Adv., 2015, 5, 18342-18351.

20 S. Vadahanambi, S. H. Lee, W. J. Kim and I. K. Oh, Environ. Sci. Technol., 2013, 47, 10510-10517.

21 W. J. Han, L. Ren, L. J. Gong, X. Qi, Y. D. Liu, L. W. Yang, X. L. Wei and J. X. Zhong, ACS Sustainable Chem. Eng., 2014, 2, 741-748. 
22 P. Wang, B. B. Huang, X. Y. Zhang, X. Y. Qin, H. Jin, Y. Dai, Z. Y. Wang, J. Y. Wei, J. Zhan, S. Y. Wang, J. P. Wang and M. H. Whangbo, Chem.-Eur. J., 2009, 15, 1821-1824.

23 P. Wang, B. B. Huang, X. Y. Qin, X. Y. Zhang, Y. Dai, J. Y. Wei and M. H. Whangbo, Angew. Chem., Int. Ed., 2008, 47, 7931-7933.

24 B. W. Ma, J. F. Guo, W. L. Dai and K. N. Fan, Appl. Catal., B, 2013, 130, 257-263.

25 Y. Zhuang, J. H. Sun and M. Y. Guan, J. Alloys Compd., 2016, 662, 84-88.

26 Y. Li, W. Q. Cui, L. Liu, R. L. Zong, W. Q. Yao, Y. H. Liang and Y. F. Zhu, Appl. Catal., B, 2016, 199, 412-423.

27 X. Y. Pan and Z. G. Yi, ACS Appl. Mater. Interfaces, 2015, 79, 27167-27175.

28 X. Huang, X. Y. Qi, F. Boey and H. Zhang, Chem. Soc. Rev., 2012, 41, 666-686.

29 Y. Y. Sun, W. H. Zhang, D. S. Li, L. Gao, C. L. Hou, Y. H. Zhang and Y. Q. Liu, J. Alloys Compd., 2015, 649, 579-584.

30 J. J. Yang, D. M. Chen, Y. Zhu, Y. M. Zhang and Y. F. Zhu, Appl. Catal., B, 2017, 205, 228-237.

31 D. Li, M. B. Muller, S. Gilje, R. B. Kaner and G. G. Wallace, Nat. Nanotechnol., 2008, 3, 101-105.

32 Y. H. Liang, S. L. Lin, L. Liu, J. S. Hu and W. Q. Cui, Appl. Catal., B, 2015, 164, 192-203.

33 P. Wang, T. S. Ming, G. H. Wang, X. F. Wang, H. G. Yu and J. G. Yu, J. Mol. Catal. A: Chem., 2014, 381, 114-119.

34 L. Liu, L. Ding, Y. G. Liu, W. J. An, S. L. Lin, Y. H. Liang and W. Q. Cui, Appl. Catal., B, 2017, 201, 92-104.

35 C. J. Miller, H. J. Yu and T. D. Waite, Colloids Surf., A, 2013, 435, 147-153.

36 Z. Y. Zeng, Z. Y. Yin, X. Huang, H. Li, Q. Y. He, G. Lu and F. Boey, Angew. Chem., Int. Ed., 2011, 50(47), 11093-11097.
37 S. T. Zhong, W. Jiang, M. Han, G. Z. Liu, N. Zhang and Y. Lu, Appl. Surf. Sci., 2015, 347, 21.

38 N. Zhang, M. Q. Yang, S. Q. Liu, Y. G. Sun and Y. J. Xu, Chem. Rev., 2015, 115, 10307-10377.

39 M. Nawaz, W. Miran, J. Jang and D. S. Lee, Appl. Catal., B, 2017, 203, 85-95.

40 R. Ccorahua, O. P. Troncoso, S. Rodriguez, D. Lopez and F. G. Torres, Carbohydr. Polym., 2017, 171, 68-76.

41 S. S. Nanda, M. J. Kim, K. S. Yeom, S. S. An, H. Ju and D. K. Yi, TrAC, Trends Anal. Chem., 2016, 80, 125-131.

42 W. S. Si, W. Lei, Q. L. Hao, X. F. Xia, H. Zhang, J. Li and Q. H. Li, Electrochim. Acta, 2016, 212, 784-790.

43 J. J. Wu, D. Zhang, Y. Wang and B. R. Hou, J. Power Sources, 2013, 227, 185-190.

44 L. Liu, L. Ding, Y. G. Liu, W. J. An, S. L. Lin, Y. H. Liang and W. Q. Cui, Appl. Catal., B, 2016, 183, 133-141.

45 X. R. Hong, R. P. Gang, H. Jian, R. Fang, R. L. Zhen and S. Z. Feng, Carbohydr. Polym., 2016, 138, 222-228.

46 J. J. Yang, D. M. Chen, Y. Zhu, Y. M. Zhang and Y. F. Zhu, Appl. Catal., B, 2017, 205, 228-237.

47 C. F. Mu, Y. Zhang, W. Q. Cui, Y. H. Liang and Y. F. Zhu, Appl. Catal., B, 2017, 212, 41-49.

48 Z. Y. Zhang, F. Xiao, Y. L. Guo, S. Wang and Y. Q. Liu, ACS Appl. Mater. Interfaces, 2013, 5, 2227-2233.

49 M. Wen, M. Z. Cheng, S. Q. Zhou, Q. S. Wu, N. Wang and L. Y. Zhou, J. Phys. Chem. C, 2012, 116, 11702-11708.

50 J. Wang, Z. M. Chen and B. L. Chen, Environ. Sci. Technol., 2014, 48, 4817-4825.

51 G. Q. Luo, X. J. Jiang, M. J. Li, Q. Shen, L. M. Zhang and H. G. Yu, ACS Appl. Mater. Interfaces, 2013, 5(6), 2161-2168. 